\section{Active surveillance of adverse events following ChAdOx1 nCoV-19 immunization in geriatric population: a prospective multi-centric study from Jaipur, Rajasthan, India}

\author{
Anil Bhandari, ${ }^{1}$ \\ Surya Pratap Singh Tiwari, ${ }^{2}$ \\ Monika Rathore, ${ }^{3}$ Lokendra Sharma, ${ }^{1}$ \\ Smita Jain \\ ${ }^{1}$ Department of Pharmacology, SMS \\ Medical College, Jaipur; ${ }^{2}$ SMS Medical \\ College, Jaipur; ${ }^{3}$ Department \\ of Community Medicine, SMS Medical \\ College, Jaipur; ${ }^{4}$ Department \\ of Mathematics, JECRC University \\ of Jaipur, Jaipur, Rajasthan, India
}

\section{Abstract}

India launched its coronavirus disease 2019 (COVID-19) vaccination drive starting with healthcare workers. The aim of the study was to evaluate adverse events following immunization (AEFI) amongst the Geriatric population associated with two doses of ChAdOx1 nCoV-19 vaccine. We also evaluated association of AEFI according to gender and elderly age groups.

An observational study, conducted among 437 individuals vaccinated at multiple community healthcare centers in Jaipur, of AEFIs associated with both doses of ChAdOx1 nCoV-19 vaccine, via telephonic interviews on the day of vaccination-Day 0 , Day 7 and Day 15 from vaccination.

463 vaccinated individuals who responded for first dose AEFIs, and 437 (94.3\% 437/463) responded to the telephone interview regarding the second dose. Of these, $5.5 \%(24 / 437)$ reported AEFIs for the second dose. Among 60 respondents who reported AEFI (both doses) fever (26) and fatigue (22) were the most reported systemic AEFI. Local AEFIs were injection site soreness (23). The AEFIs (both systemic and local) in respondents mostly lasted for 1-2 days. AEFI reported by respondents in the age group 6070 years was higher than those above 70 years. Female respondents were associated with higher AEFI than the males.

The AEFIs of both the doses were observed in the first 2 days predominantly. Symptoms were minor, short lived and selflimiting. No serious adverse events attributable to vaccines were reported in our study. Adverse event following immunization is independent of gender and age distribution for both the doses.

\section{Introduction}

On 11 March 2020, the World Health Organization (WHO) declared the novel coronavirus disease 2019 (COVID-19) outbreak as a global pandemic. ${ }^{1}$ Till September 27, 2021, India recorded more than 33.8 million cases and 448,000 deaths from the day the coronavirus pandemic hit in December 2019. ${ }^{2}$ India rolled out its mass vaccine campaign against COVID-19 on January 16, 2021, with the most used vaccine i.e., ChAdOx1nCoV-19. Manufactured by Serum Institute of India in partnership with OxfordAstraZeneca, it consists of a replication-deficient chimpanzee adenoviral vector ChAdOx1, containing the SARS-CoV-2 structural surface glycoprotein antigen (spike protein; $\mathrm{nCoV}-19$ ) gene. ChAdOx1 nCoV-19 vaccination course consists of two separate doses of $0.5 \mathrm{ml}$ each. Initially the gap between the two doses of ChAdOx1 nCoV19 was 28 days. This gap was increased to 68 weeks in March 2021, which was further increased to 12-16 weeks in May 2021.As of 1 October 2021, over 89 million COVID-19 vaccination, which represents approximately $64 \%$ of the population. ${ }^{3}$

When people began to feel some hope in summer 2021 that the pandemic could recede to the background, the Delta variant emerged. First identified in India in late 2020, Delta swept rapidly and accounting for more than $99 \%$ of COVID-19 cases and leading to an upsetting increase in hospitalizations ${ }^{4} \mathrm{R}_{0}$ value (Indicates how contagious an infectious disease is) of delta variant was found to be higher $\left(\mathrm{R}_{0}\right.$ of 5.08) of the any ancestral strains $\left(\mathrm{R}_{0}\right.$ of 2.79) of SARS-CoV$2 .^{5}$ Hence, the people who were reluctant from vaccination were more in danger and super spreader of COVID in places with low vaccination rates. With increase in vaccination rates, many cases of adverse effects have been reported, including those that are common and less severe and those that are more sever and rare like vaccine-induced immune thrombotic thrombocytopenia and hemorrhagic events. ${ }^{6,7}$

Providing detailed information on adverse effects is essential for communicating the risks associated with vaccinations and to educate the general population and encourage them to participate in vaccination. However, the data currently available regarding AEFIs of ChAdOx1 nCoV-19 vaccine and comparison between AEFIs of the two doses are insufficient. Therefore, we identified the adverse effects associated
Correspondence: Anil Bhandari and

Lokendra Sharma, Department of Pharmacology, SMS Medical College, Jaipur 302004, Rajasthan, India.

E-mail: anil.bhandari@gmail.com ; drlokendra29@gmail.com

Key words: COVID-19 vaccine ChAdOx1nCoV-19; adverse events following immunization.

Acknowledgments: we are grateful to Community Healthcare Centers providing valuable data and 'Swastik Ethics

Committee' for approving our study.

Contributions: $\mathrm{AB}$, study concept and design, definition of intellectual content, literature search, clinical studies, data acquisition and analysis, statistical analysis, manuscript preparation, editing and review, guarantor; SPTS, study concept and design, definition of intellectual content, literature search, clinical studies, data analysis, manuscript preparation, editing and review; MR, study design, definition of intellectual content, statistical analysis, manuscript editing and review; LS, definition of intellectual content, manuscript preparation, editing and review; SJ, study design, data analysis, statistical analysis.

Received for publication: 12 June 2021. Revision received: 7 January 2022. Accepted for publication: 12 January 2022.

This work is licensed under a Creative Commons Attribution-NonCommercial 4.0 International License (CC BY-NC 4.0).

${ }^{\circ}$ Copyright: the Author(s), 2021

Licensee PAGEPress, Italy

Geriatric Care 2021; 7:9908

doi:10.4081/gc.2021.9908

ChAdOx1 nCoV-19 and drew a comparison between AEFIs from the first dose and the second, to provide a real-life data which would form a basis for ensuring safety during the future national vaccination against Covid-19 and to get over the vaccine hesitancy.

\section{Objective}

The objective was to draw a comparison between the AEFIs associated with the first and second dose of ChAdOx $1 \mathrm{nCoV}-19$ vaccine among vaccinated individuals above 60 years of age.

\section{Materials and Methods}

\section{Study design and population}

An observational study was conducted at multiple community vaccination centers in 
Jaipur. The study subjects were above 60 years of age, who had completed the first and second dose of ChAdOx1 nCoV-19 vaccine.

\section{Data collection and adverse reaction reporting system}

The vaccinated participants were contacted via landline by one of the authors. Telephone interview was conducted based on a questionnaire, which was customized based on Pharmacovigilance Performa provided by a WHO Collaborating center Pharmacovigilance Programme of India (PvPI) - Indian Pharmacopoeia Commission, Ghaziabad. Telephonic calls were made on day of second dose of ChAdOx1 nCoV-19 vaccine i.e., Day 0, seven days after vaccination i.e., Day-7, fifteen days after vaccination i.e., Day-15. All the AEFI were uploaded in 'Vigiflow' (a web-based individual case safety report (ICSR) management system that is available for use by national pharmacovigilance centers of the WHO Programme for International Drug Monitoring) at Adverse Drug Reaction Monitoring Center in SMS Medical College, Jaipur (Rajasthan).

\section{Statistical analysis}

Data was analyzed using Microsoft Excel, Minitab (a statistical software) and online statistical tool was used for performing the chi-square test, to test the association between the gender and AEFI status and between the age group and respondent's AEFI. The respective mean, mode, standard deviation and percentage were calculated for the survey data.

\section{Results}

Out of 463 vaccinated individuals who responded for first dose AEFIs, and 437 $(94.3 \%$ 437/463) responded to the telephone interview regarding the second dose. However, we were not able to report some individuals due to non-availability of the receiver. The mean age of the respondents was 67.5 years $(\mathrm{SD}=6.5$, Range $=60-94)$. $56.75 \%$ were male $(248 / 437)$ and $43.2 \%$ were female (189/437). Out of the respondents, 5.5\% (24/437) reported minor AEFI and none reported severe AEFI.

Among 60 respondents who reported AEFI (both doses): fever (26) and fatigue (22) were the most reported systemic AEFI. Injection site soreness (23) was the most reported local AEFI (Table 1) (Figure 1) The $r$ value (Pearson correlation coefficient) for the relationship between the data of first and second dose with respect to the different symptoms is 0.834 and the $\mathrm{P}$ value is 0.039. 26 Respondents took medications for their symptoms while resting at home, which included over-the-counter medicines like paracetamol for fever and fatigue, one of the respondents consulted to a General Practitioner (GP) for post-vaccination symptoms.
In most of the respondents, symptoms lasted for more than 24 hours. The AEFIs (both systemic and local) in respondents mostly lasted for $1-2$ days $(66.7 \%$; 40/60) from the day of vaccination (Figure 2) AEFI reported by respondents in the age group 60-70 years (44) was higher than those above 70 years (16) (Tables 2 and 3) (Figure 3 ). The $P$ value for the association between Age group and respondent AEFI is 0.196. Female respondents (35) were associated with higher AEFI than the males (25).

\section{Discussion}

In this prospective, community-based study conducted at multiple community healthcare center in Jaipur, we have investigated adverse events following immunization (AEFIs) associated with first and second dose of ChAdOx1 nCoV-19 vaccine. In

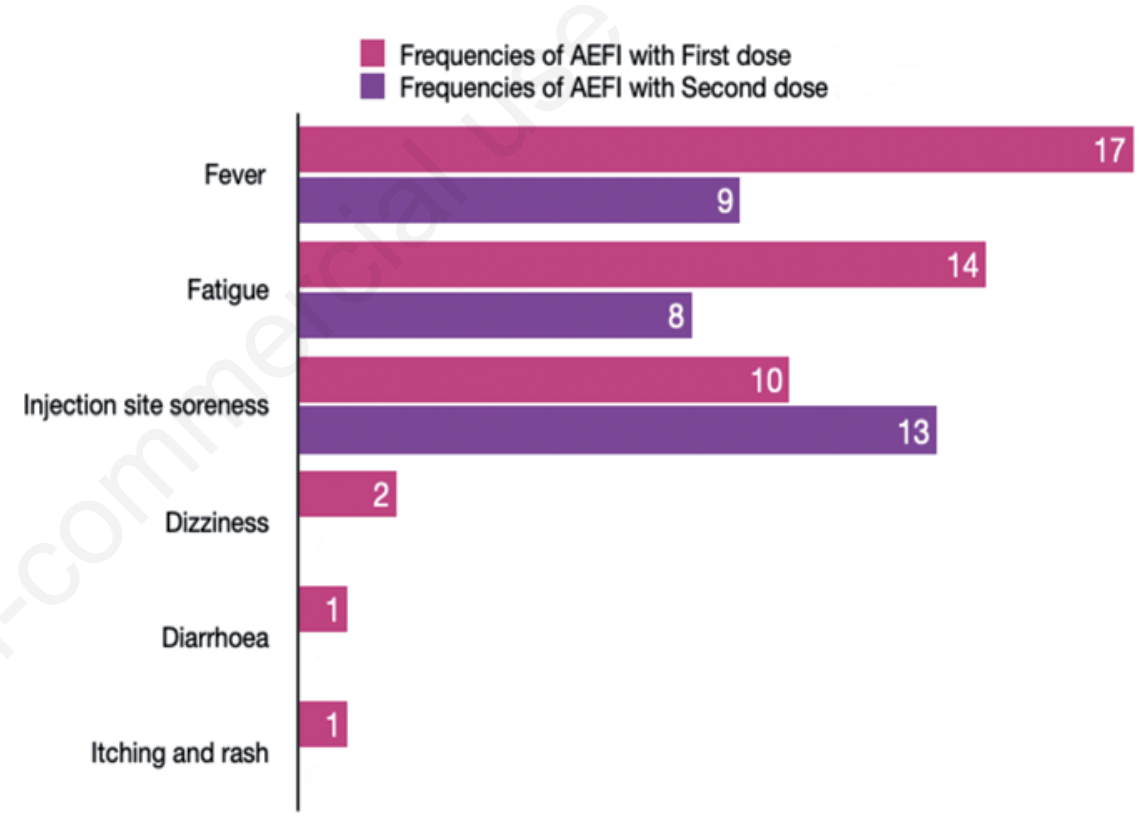

Figure 1. Frequencies of adverse events following ChAdOx1 nCoV-19 immunization (AEFI).

Table 1. Adverse events following ChAdOx1 nCoV-19 immunization reported by the study population.

\begin{tabular}{|c|c|c|c|c|}
\hline Symptoms & \multicolumn{2}{|c|}{ Systemic symptoms with first dose } & \multicolumn{2}{|c|}{ Systemic symptoms with second dose } \\
\hline Fatigue & 14 & $3.02 \%$ & 8 & $1.83 \%$ \\
\hline Fever & 17 & $3.67 \%$ & 9 & $2.05 \%$ \\
\hline Dizziness & 2 & $0.43 \%$ & 0 & $0 \%$ \\
\hline Diarrhea & 1 & $0.21 \%$ & 0 & $0 \%$ \\
\hline \multicolumn{3}{|c|}{ Local site symptoms with first dose } & \multicolumn{2}{|c|}{ Local site symptoms with second dose } \\
\hline Injection site soreness & 10 & $2.15 \%$ & 13 & $2.97 \%$ \\
\hline Itching and rash & 1 & $0.21 \%$ & 0 & $0 \%$ \\
\hline
\end{tabular}


our study, overall common adverse events after the first and second dose of the ChAdOx1 nCoV-19 vaccine were fever, fatigue, injection site soreness. No serious AEFI attributable to vaccines were reported and all respondents who developed AEFIs, improved within 2 days $(66.7 \% 40 / 60)$ of vaccination. The rate of adverse events showed a declining trend after 2 days of vaccination. Symptoms were mild in severity and short-lived. ChAdOx1 nCoV-19, thus have minor, self-limiting, and tolerable AEFIs associated with both, the first and the second dose. This supports the findings of phase 1 and phase $2 / 3$ trial of ChAdOx 1 nCoV-19 vaccines wherein most recipients reported with non-serious AEFI.

In our study, $7.8 \%$ of the study population reported AEFIs with first dose and $5.5 \%$ with the second dose. These findings are in agreement with the findings of Phase 2/3 Safety trial of ChAdOx 1 nCoV-19 where fewer adverse events were reported after the boost vaccination than after the prime vaccination. ${ }^{8}$ Also, we found that for respondents in the age group 60-70 years, AEFIs reported were $8.7 \%$ with the first dose and $6.4 \%$ with the second dose, and in those above 70 was $6.2 \%$ with the first dose and $3.9 \%$ with the second dose, which concur findings of phase $2 / 3$ safety trial of ChAdOx1 nCoV-19 where reactogenicity reduced with increasing age. However, in our study the adverse events were reported at much lower frequencies in comparison to the Phase 2/3 trial of the ChAdOx $1 \mathrm{nCoV} 19$ vaccine where $73 \%$ in 56-69 year group and $61 \%$ in the 70 years and older group, reported adverse events. Higher number of minor AEFI were reported by female respondents than the males, irrespective of the age group. However, we did not find any supporting evidence of high reactogenicity in females, but this might be due to the influence of various sociodemographic variables in our survey data.

The Ministry of Health and Family
Welfare noted that as of, 30th November 2021 1,240,157,719 vaccine doses had been administered. Of these, 788,635,410 were first doses and 451,522,309 second dose. ${ }^{9}$ As on 15th June 2021, the number of deaths reported following COVID-19 vaccination was $0.0002 \%$ of 235 million doses administered. ${ }^{10}$ AEFI data in India showed that there is a very minuscule but definitive risk of thromboembolic events. However, no such serious and thromboembolic event was reported by respondents in our study group.

\section{Limitation}

The major limitation of our study is the relatively small sample size to assess serious/rare AEFI which could be attributed to the location of the multiple healthcare center which were remote and had a relatively less inflow of population. Since, the study was conducted only on specific age group, reporting bias is possible. Other limitations of our study could be recall bias as follow up interviews were carried out via telephone.

\section{Conclusions}

Only 7.7\% respondents experienced adverse effect after the first and 5.5\% after the second dose of ChAdOx1 nCoV-19 vaccine. Though all were mild and short-lived.

\section{Respondents with AEFIs with first dose}

Respondents with AEFIs with second dose
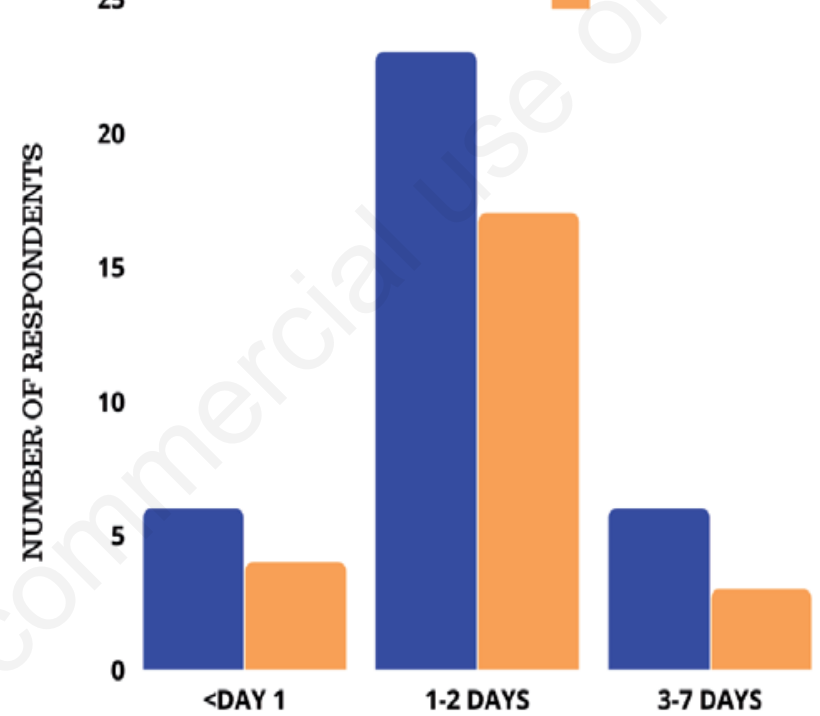

3-7 DAYS

More than 7days

\section{DURATION OF POST-VACCINATION SYMPTOMS}

Figure 2. Duration of postvaccination symptoms with ChAdOx1 nCoV-19 vaccine. AEFI, adverse events following ChAdOx1 nCoV-19 immunization.

Table 2. Adverse events following ChAdOx1 nCoV-19 immunization reported in different age groups.

\begin{tabular}{|c|c|c|c|c|}
\hline Age group & $\begin{array}{l}\text { Respondents with AEF } \\
\text { with first dose }\end{array}$ & $\begin{array}{l}\text { Respondents with no } \\
\text { AEFI with first dose }\end{array}$ & $\begin{array}{l}\text { Respondents with AEFI } \\
\text { with second dose }\end{array}$ & $\begin{array}{l}\text { Respondents with no AEFI } \\
\text { with second dose }\end{array}$ \\
\hline $60-65$ & 18 & 155 & 12 & 154 \\
\hline $65-70$ & 8 & 116 & 6 & 110 \\
\hline $70-75$ & 7 & 93 & 4 & 90 \\
\hline $75-80$ & 3 & 36 & 1 & 34 \\
\hline $80-85$ & 0 & 16 & 1 & 15 \\
\hline $85-90$ & 0 & 8 & 0 & 8 \\
\hline \multirow[t]{2}{*}{$90-95$} & 0 & 3 & 0 & 2 \\
\hline & 36 & 427 & 24 & 413 \\
\hline
\end{tabular}

AEFI, adverse events following ChAdOxl nCoV-19 immunization. 
Table 3. Aggregate age wise distribution of respondents regarding adverse events following ChAdOx1 nCoV-19 immunization associated with both doses.

\begin{tabular}{lccc} 
Age group & Respondents with AEFI & Respondents without AEFI & No respondents \\
$60-65$ & 30 & 309 & 339 \\
$65-70$ & 14 & 226 & 240 \\
\hline $70-75$ & 11 & 183 & 194 \\
$75-95$ & 5 & 122 & 127 \\
\hline
\end{tabular}

AEFI, adverse events following ChAdOxl nCoV-19 immunization.

Respondents with AEFIs with first dose

Respondents with No AEFIs with first dose

300

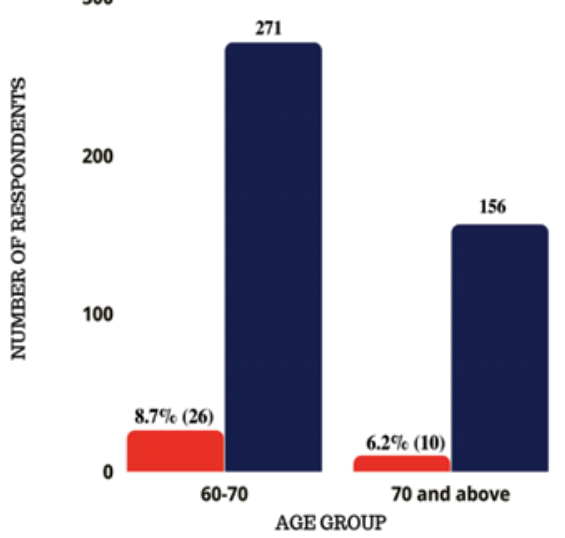

Respondents with AEFIs with second dose

Respondents with No AEFIs with second dose

300

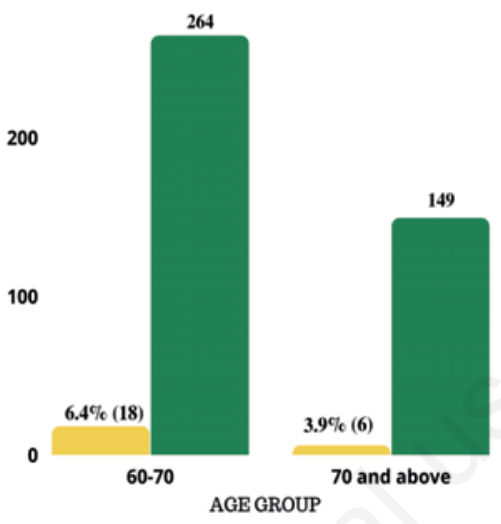

Figure 3. Age wise distribution of adverse events following immunization during monitored period after vaccination. AEFI, adverse events following ChAdOx1 nCoV-19 immunization.

On applying Chi-Square test we conclude that there is no existence of evidence that depicts association between gender and AEFI status. Also, no evidence exists regarding association between age group and response of AEFI status for both the doses of the vaccine as the $\mathrm{P}$ value is greater than 0.05 . Thus, it could be concluded that adverse event following immunization is independent of gender and age distribution at the time of first and second dose.

We also found a relation between the data of 'first and second dose' with respect to the different symptoms, which indicates that the same symptoms will be observed after the second dose also. Since the P value is 0.039 , we conclude that the relationship exists for the population also.

The AEFIs of both the doses were observed in the first 2 days predominantly.
Symptoms were minor, self-limiting, and had a lower reactogenicity profile with both doses of ChAdOx1nCoV-19. No serious adverse events attributable to vaccines were reported in our study.

\section{References}

1. Cucinotta D, Vanelli M. WHO declares COVID-19 a pandemic. Acta Biomed 2020;91:157-60.

2. WHO. Coronavirus dashboard. Available from: https://covid19.who. int/table Accessed: 1 October 2021.

3. Ministry of Health and Family Welfare. Covid vaccination update - day 59; 1 October 2021. Available from: https:// pib.gov.in/Press ReleasePage.aspx?
PRID=1760107 Accessed: 15 October 2021.

4. Yale Medicine. 5 things to know about delta variant; 21 December 2021. Available from: https:/www.yalemedicine.org/news/5-things-to-know-deltavariant-covid Accessed: 25 December 2021.

5. Liu Y, Rocklöv J. The reproductive number of the Delta variant of SARSCoV-2 is far higher compared to the ancestral SARS-CoV-2 virus. J Travel Med 2021;28:taab124.

6. Menni C, Klaser K, May A, et al. Vaccine side-effects and SARS-CoV-2 infection after vaccination in users of the COVID Symptom Study app in the UK: a prospective observational study. Lancet Infect Dis 2021;S1473-3099(21) 00224-3.

7. Long B, Bridwell R, Gottlieb M. Thrombosis with thrombocytopenia syndrome associated with COVID-19 vaccines. Am J Emerg Med 2021; 49:58-61.

8. Ramasamy MN, Minassian AM, Ewer KJ, Flaxman AL, Folegatti PM, Owens $\mathrm{DR}$, et al. Safety and immunogenicity of ChAdOx1 nCoV-19 vaccine administered in a prime-boost regimen in young and old adults (COV002): a single-blind, randomized, controlled, phase 2/3 trial. Lancet 2021;396: $1979 \mathrm{e} 1993$.

9. Ministry of Health and Family Welfare. India's cumulative vaccination coverage crosses 124 crore landmark milestone; 30 November 2021. Available from: https://pib.gov.in/PressRelease Page.aspx?PRID=1776592

10. Ministry of Health and Family Welfare. COVID19 vaccination: myths vs facts; 15 June 2021. Available from: https://pib.gov.in/PressReleasePage.asp $\mathrm{x}$ ?PRID=1727196 